\title{
Computing the temporal intervals by making a Throne-Morris worm- hole from a Kerr black hole in the context of $f(R, T)$ gravity
}

\author{
Aruna Harikant ${ }^{1}$, Sanjeevan Singha Roy ${ }^{2}$, Deep Bhattacharjee ${ }^{3}$ \\ ${ }^{1}$ Department of Physics, Indian Institute of Technology, Mandi, Kamand, Himachal Pradesh, India \\ ${ }^{2}$ Department of Physics, Birla Institute of Technology, Mesra, Jharkhand, India \\ ${ }^{3}$ Departmental In-Charge, AATWRI-R\&D directorate of electro-gravitation simulation and propulsion \\ laboratory, Bhubeneshwar, Odisha, India \\ ${ }^{3}$ Research Mentor, Research Convention, Chandigarh, Punjab, India
}

\begin{abstract}
In the paper we will proceed towards taking the larger root of $\Delta$ and make it equal to zero to remove the event horizon of a Kerr black hole (BH) in Boyer-Lindquist coordinates with a prevalent ring type singularity that can be smoothen by a tunneling approach of a spherinder thereby proceeding safely towards the Cauchy horizon with the deduced intervals computed in detail for the time travel in the Throne-Morris wormhole (WH) approach under $f(R, T)$ gravity without the presence of any exotic matter at the WH mouth thereby preserving the asymptotically solutions of flaring out conditions and mouth opening during the course of the journey through the Einstein-Rosen bridge. An approach has been organized in the paper in which not only time travel is possible without exotic matter but also time travel is flexible to past and future in the Einstein's universe by eliminating all sorts of paradoxes by $d-4$ spatial sheath through 2D approach of temporal dimensions.
\end{abstract}

Keywords - Throne-Morris Wormhole; Kerr Black Hole; Naked Singularity; $f(R, T)$ gravity; Spherinder; Exotic Matter; Time Travel; Closed Timelike Curves; $d-4$ Spatial Sheath; $2 D$ Temporal Dimensions

\section{Introduction}

Shattering the absolute concept of the rigid time of Newton [1], Einstein introduced to us the domain of the relative time [2] where time can be fast, slow or even stop at the speed of the light. This dilation is the key to the various solutions of the Einstein field equations from which derived the concept of the collapsing stellar objects like the black hole $(\mathrm{BH})[3,4,5]$. Inspite of the technological and engineering hindrances, the concept of time travel has fascinated humans more than a centuries which all started from the relative nature of the time as shown by Einstein in his equations of general theory of relativity. Various novels, fictional like H.G. well's The Time Machine [6], Carl Sagan's Contact [7] or even the sci-fi movies of Christopher Nolan's Interstellar [8] not only depicted the logic behind time travel but also shown the legitimate reasoning and visualization of the WHs where the time travel can take place. The first solution of the BH mechanics has been given by Schwarzschild in the form of the metric shortly after Einstein gave his equations of general relativity, which depicts a non-spin $\mathrm{BH}$ with a coordinate singularity [9]. Then after that Einstein along with his colleague Nathan Rosen gives the tantalizing idea of a hypothetical bridge that could be possible at one end of a $\mathrm{BH}$ to the other end of a $\mathrm{BH}$ in the form of a temporal tunnel called the Einstein-Rosen bridge [10]. The special property of this bridge is that, it is completely devoid of any horizon and is singularity free. Since then numerous solutions have been provided in the form of various metrics of that bridge that mathematically fascinates time travel. Hawking and Ellis worked on this and Ellis had gave the solution of a drainhole that behaves as a wormhole $[11,12]$. As days passed by a paper with a very detailed calculation 
has been chalked out by Throne and Morris showing us the possibility of a traversal time tunnel through which one can move from the horizon to the anti-horizon [13]. The Kerr solution that exists have always predicted the white holes at the end of the BHs but its extension to a Einstein-Rosen bridge (E-R) has not been provided specifically since it has a horizon and a ring singularity [14]. moreover, a traveler can pass through the ring singularity but he might die due to the tidal attraction inside the $\mathrm{BH}$. Throne and Morris have also explained in their 1988 paper, that, time travel always comes with a price and that is the requirement of the exotic matter or $\zeta \gg \rho^{2} c$ which asserts that tension is always greator than material matterdensity multiplied by the speed of the light which hints new constraints in the form of something 'exotic' in nature to prevent the WH from being collapsing [15].

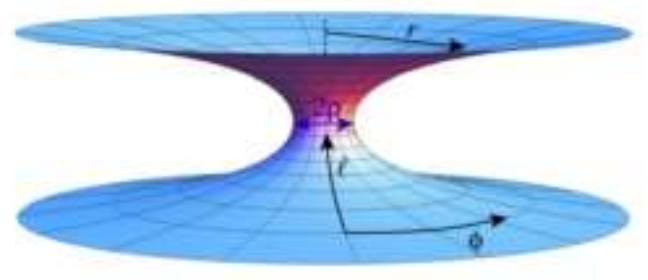

Figure 1. Embedding diagram of a WH consisting with the equation

$r(\ell)=\left\{\begin{array}{r}\rho, \text { for the wormhole interior }|\ell| \leq \alpha \\ |\ell|-\alpha+\rho, \text { for the wormhole exterior }|\ell|>\alpha\end{array}\right.$ depicting the detailing of the WH structure.

Ref. [16]

This is however, also a source of uncannyness whether the anti-horizon of a white hole could act in an affine way so as to eject the time traveler to another domain of time or another universe. It should be stated that both the WHs end i.e., white whole and the Cauchy horizon is unstabilized by small perturbations and if any outgoing particle comes in contact with their horizon then they will be Doppler blue shifted to such an huge magnitude that they will collapse the entire tunnel [17, 18, 19]. Therefore its difficult to obtain a stable theory of a particle trajectory through a WH although we have tried our best to provide a solution in terms of $f(R, T)$ modified Throne-Morris geometry that doesn't require any sort of exotic matter dependable upon certain parameters of the throat function of WHs [20]. Space-time can generally be divided into two regimes, the nonchronal regions where there are CTC's or the chronal regions devoid of any CTC's. a Cauchy horizon can be stated as a future chronal horizon where if any null rays followed into the future have no past end points. And if any arbitrarily advanced civilization tries to constant a time machine then they will choose the chronologically compact chronal horizons rather than uncompact regimes. This paper takes into account the chronologically compact zones such as singularity that may be utilized for the time travel. However we have also shown that the energy conditions remains valid under all circumstances yielding a positive stressenergy tensor $T_{\mu \nu} \geq 0$ which includes the strong energy conditions (SEC), dominant energy conditions (DEC), weak energy conditions (WEC), null energy conditions (NEC), average null energy conditions (ANEC) being zero or non-negative everywhere throughout the WH geometry [20]. Many physicists agued that in the Einstein's universe the stress-energy must be violated to provide a suitable asymptotically flat flaring out conditions of the WH mouths. Stephen W. Hawking has further shown in his celebrated paper chronology protection conjecture [17] that not only the energy conditions being violated but also nature would impose a chronal agent that forbids time travelling beyond Plank's length. This forbidden mechanism is deeply worrying and so, Kip S. Throne showed in a later paper [19] that if chronology can be protected then there must be hope that it is weakly protected rather than strongly protected giving us a little hope for time travelling. To mention another thing is that, the Einstein's universe has always showed us solutions of a temporal travelling trajectory in a uni-directional way that is from past to future but not from future to past. This has been done to prevent causality violations or any sorts of paradoxes like the grandfather's paradox [21], 
Polchinsky paradox [22], bootstrap paradox [23]. Through this paper we will show the mechanics that how those paradoxes could be resolved by introducing a $d-4$ spatial sheath that will cover the time traveler when he/she will travel through time providing a superficially one way visualization by which only the time traveler can see his own past or future upto the specified limits as and where appropriate but the observer can in no way see the time traveler being warped in the $d-4$ sheath or membrane. Because time itself takes a 2D [24] form as we will compute in this paper with various intervals between them. We will start the methodology by giving a beautiful example of Stephen W. Hawking's time travelling party where when no one's (or presumable time travelers) arrived, he joyfully quoted "if time travelling is possible then where are the hoards of time travelers from the future?" which compels us to believe that he is absolutely right, but we will shift our focus to a more modified versions of gravity where things are different a little bit.

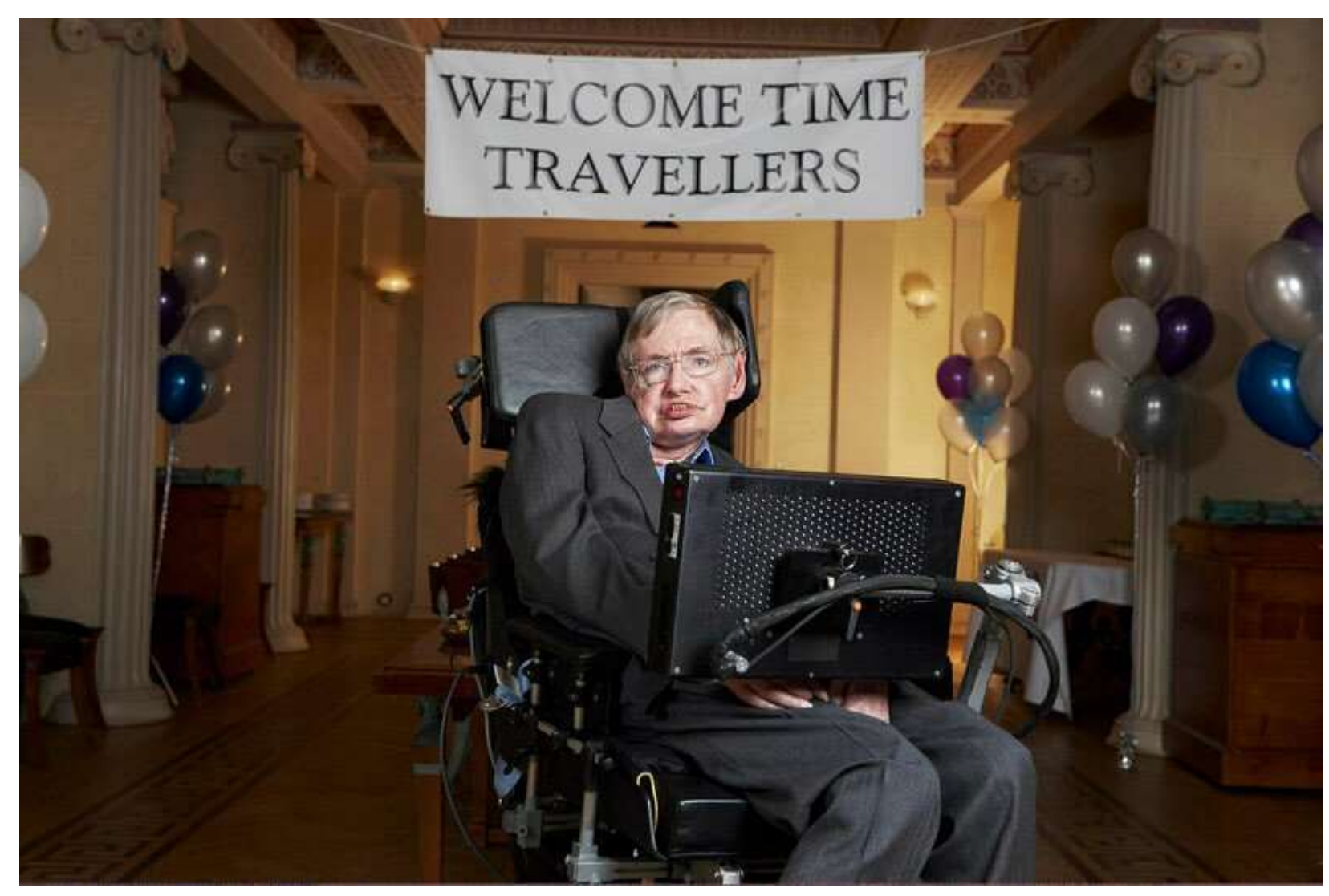

Figure 2. Stephen W. hawking had given a party for the time travelers after publishing his revolutionary paper on "Chronology protection" when he asserted that nature must be equipped with a chronal agent for the chronology protection mechanism to forbid travelling through time. His party also raises the question that "where are the hoards of time travelers from future? If nobody attended his party!" with brevity and mathematics he had shown how chronology is protected thereby "making the history safe for historians" in his own language. However, Kip. S. Throne in his paper pointed out the fact that with each refocusing and defocusing of the null rays or vacuum polarizations in each circuits from one mouth of the WH to the other, the contributing stress-energy tensor $T^{\mu \nu}$ (which according to Hawking must be negative i.e., $\oint T_{\mu \nu} l^{\mu} l^{\nu} \ll 0$ can be renormalized through the splitting point relations, with each consecutive foliations (taking units $G=c=$ 1) and Plank's length $P_{l}$ such that $P_{l}=\sqrt{\hbar}$ with the spatial parameter $\mho$ that is sharply varying with the refocusing parameter as $(\Delta>1)$ and defocusing parameter $(\Delta<1)$ provides the relation $T_{\mu \nu}=\frac{\sqrt{\Delta}}{6 \pi^{2}} \frac{P_{l}^{2}}{\mho^{3}}\left(2 k_{\mu} l_{v}+2 k_{\nu} l_{\mu}+k_{\mu} k_{v}+l_{\mu} l_{v}+g_{\mu \nu} l^{\alpha} k_{\alpha}\right)$ where the pile up radiation backreacts through the WH mouth of each circuit as $\frac{\lambda}{2 D}$ as the vacuum polarization distortion which could be too weak to protect the chronology, when the time interval $\Delta t=P_{l}$ through the horizon passage in each region, where time itself 
doesn't make any sense like the classical limit (contrary to the large WH we have discussed having the mouth as 1 light year and the relative circumference $>10^{4}$ solar masses for the tidal distortions not to take effect on the traveler) where if the hold is feasible, then quantum gravity will smoothen out the divergences where the metric divergence being too small $\epsilon g_{\mu \nu}^{V P}$ with VP as vacuum polarizations giving the relation, $\epsilon g_{\mu \nu}^{V P} \sim \frac{P_{l}}{D} \sim 10^{35}$ meter where $D$ the distance between two commoving mouths of WH in the hypersurface being taken as a small limit of $\sim 1$ meter in case of $n \sim \infty$ turns, as given by the equation $\epsilon g_{\mu \nu}^{V P} \sim \frac{P_{l}^{2}}{D \Delta t} \sum_{n}\left(\frac{\lambda}{2 D}\right)^{n-1}$ to unprotect chronology thereby making the time travel feasible at the Plank regime. Ref. [17, 19, 25].

Its specific that we will construct the WH from the blowing out event horizon of a Kerr BH and smoothen or tunneling its ring singularity by means of a higher analogue of cylinder called the spherinder [26] where the present itself takes upon conjugate values as and when necessary. The paper has been constructed in the following way: section II describes the details of the Kerr BH solutions in Boyer-Lindquist coordinates thereby describing both of its horizons, ergosurface and symmetry with a specific focus on the ring singularity. Then the particle motion around the $\mathrm{BH}$ has been provided with the Carter constant along with the gravitational time dilation via the frame dragging effect and Lense-Thirring precessions. After that we have taken the larger root of $\Delta=0$ denoting the absence of event horizons thereby making a naked singularity. The event horizon problem can be solved but still remains the singularity problem which we will try to smoothen out via spherinder tunneling effect in section VI. Section III then describes the detailed properties of the ThroneMorris WH metric by deducing its components and analyzing it as appeared in their 1988 publications which imposes an additional problem that the WH requires an exotic type of matter to prevent it from collapsing in which the tension force must be greater than the material density as $\zeta \gg \rho c^{2}$ which imposes the need for exotic matters. Section IV denotes the $f(R, T)$ type solutions of the Throne-Morris WH which provide new elements of the density, radial pressure, tangential pressure in such a way that exotic matters can be prohibited, thereby imposing a stable solutions than can be achievable theoretically in principle. Section $\mathrm{V}$ shows us the Kerr metric in 3-coordinate forms i.e., $\theta, \phi, t$ which computes and denotes the details of temporal intervals that is required to sheath the WH-inside from the travelers perspectives, that will show how the unique and scattered timelines represent themselves holographically in front of the time traveler where the time traveler can see his/her own past and future but they cannot see him as the $d-4$ sheath is a superficial hypermembrane of a one channel visualization only denoted by the negative symbols before the projection elements, this is important in a sense that as the traveler is totally detached from the persons/objects he is seeing behind the $d-4$ sheaths would rule out all sorts of paradoxes thereby making the "history safe for historians" as quoted by Hawking in his paper on chronology protection conjecture [17]. 9 poles have been computed and plotted from the top-bottom approach with the labeling as - possible, absurd, collapse, $\times$ and values are plotted for the throat function $b(r)=r_{0}^{2} \frac{e^{r_{0}-r}}{r}$ for variable values of $\curlywedge$ and $\alpha$ keeping the other as constant. This section assumes the time to be $2 \mathrm{D}$ for the generators of the poles and associated equations. Then section VI gets rid out of the problem of the ring singularity by tunneling it upto some specified up and down positions with the help of the higher dimensional cylinder called the spherinder that along with section VII provides the trajectories of the times in the form of a tetrad basis where it has been shown that present itself takes a complex values when the traveler is perceiving it inside the spherinder with the future being real and the past being imaginary, as futute will approach in the proper way of temporal orientation but past will be imaginary to smoothen out the notion of the singularity thereby making the traveler safe from any associated hazards [47]. Section VIII is then devoted for discussions and IX for references.

\section{Kerr Solution}


In this section, we will take a realistic metric of a $\mathrm{BH}$ as the Kerr $\mathrm{BH}$ and try to blow up the event horizon to make it a naked singularity which will be helpful in later sections of this paper where we will try to smoothen out the singularity. The Kerr line element given in Boyer-Lindquist coordinates is [27, 28],

$d s^{2}=-c^{2} d \tau^{2}=$

$-\left(1-\frac{r_{s} r}{\Sigma}\right) c^{2} d t^{2}+\frac{\Sigma}{\Delta} d r^{2}+\Sigma d \theta^{2}+\left(r^{2}+a^{2}+\frac{r_{s} r a^{2}}{\Sigma} \sin ^{2} \theta\right) \sin ^{2} \theta d \phi^{2}-\frac{2 r_{s} r a \sin ^{2} \theta}{\Sigma} c d t d \phi$

(1)

Where the oblate spheroidal coordinates are $r, \theta, \phi$ which in a similar way can be written through Cartesian coordinates as $[29,30]$,

$x=\sqrt{r^{2}+a^{2}} \sin \theta \cos \phi$

$y=\sqrt{r^{2}+a^{2}} \sin \theta \sin \phi$

$z=r \cos \theta$

(2)

Where the Schwarzschild radius is given by,

$r_{S}=\frac{2 G M}{c^{2}}$

(3)

The apparent length scales are,

$a=\frac{J}{M c}$,

$\Sigma=r^{2}+a^{2} \cos ^{2} \theta$,

$\Delta=r^{2}-r_{s} r+a^{2}$

(4)

Special points to note on Kerr BH [30],

Outer event horizon: $r_{+}=M+\sqrt{M^{2}-a^{2}}$

Inner event horizon: $r_{-}=M-\sqrt{M^{2}-a^{2}}$

Outer ergosurface: $r_{E}^{+}=M+\sqrt{M^{2}-a^{2} \cos ^{2} \theta}$

Inner ergosurface: $r_{E}^{-}=M-\sqrt{M^{2}-a^{2} \cos ^{2} \theta}$

Ring singularity: $x^{2}+y^{2}=a^{2}$ and $z=0$

Symmetry

axis:

$\theta=0, \pi$

(5)

The particle motion trajectory of invariant mass around the BH can be expressed by the equation $[31,32]$,

$-\mu^{2}=p^{\alpha} g_{\alpha \beta} p^{\beta}$

(6) 
Where $p^{\alpha}$ is the four momentum of the particle and the energy $E$, orbital angular momentum $L_{z}$ parallel to the spin of the $\mathrm{BH}$ can be given by,

$E=-p_{t}$

and

$$
L_{z}=p_{\phi}
$$

There exists another constants for motion (Carter constant $Q$ ) using the Hamilton-Jacobi theory as,

$Q=p_{\theta}^{2}+\cos ^{2} \theta\left(a^{2}\left(\mu^{2}-E^{2}\right)+\left(\frac{L_{z}}{\sin \theta}\right)^{2}\right)$

There arises four independent constants of motion which with the natural units $G=M=c=1$ shows,

$\sum \frac{d r}{d \lambda}= \pm \sqrt{R(r)}$

$\Sigma \frac{d \theta}{d \lambda}= \pm \sqrt{\Theta(\theta)}$

$\Sigma \frac{d \phi}{d \lambda}=-\left(a E-\frac{L_{z}}{\sin ^{2} \theta}\right)+\frac{a}{\Delta} P(r)$

$\Sigma \frac{d t}{d \lambda}=-a\left(a E \sin ^{2} \theta-L_{z}\right)+\frac{r^{2}+a^{2}}{\Delta} P(r)$

(9)

Where,

$\Theta(\theta)=Q-\cos ^{2} \theta\left(a^{2}\left(\mu^{2}-E^{2}\right)+\frac{L_{z}^{2}}{\sin ^{2} \theta}\right)$

$P(r)=E\left(r^{2}+a^{2}\right)-a L_{z}$

$R(r)=P(r)^{2}-\Delta\left(\mu^{2} r^{2}+\left(L_{z}-a E\right)^{2}+Q\right)$

(10)

Where $\lambda$ is a parameter producing $\frac{d x^{a}}{d \lambda}=p^{a}$, at $\mu>0$, the parameter being $\lambda$ is related with the proper time $\tau$ by $\lambda=\tau / \mu$. Due to the frame-dragging phenomena, the velocity of the test particle $v$ having an angular motion in the corotating frame $\Omega$ with the gravitational time dilation between the zero angular momentum observer at fixed $r$ and a observer being stationary away from the valued mass can be given by,

$\frac{t}{\tau}=\sqrt{\frac{\left(a^{2}+r^{2}\right)^{2}-a^{2} \Delta \sin ^{2} \theta}{\Delta \Sigma}}$

(11)

In the non-relativistic limit if $M\left(\right.$ or $\left.r_{s}\right)$ when approaches to zero, the Kerr metric for the oblate spheroidal coordinates becomes,

$g[\overrightarrow{M \rightarrow 0}]-c^{2} d t^{2}+\frac{\Sigma}{r^{2}+a^{2}} d r^{2}+\Sigma d \theta^{2}+\left(r^{2}+a^{2}\right) \sin ^{2} \theta d \phi^{2}$

The event horizon's location can be determined by the larger root of $\Delta=0$ that is when $\frac{r_{s}}{2}<a\left(G M^{2}<J c\right)$, no real valued solutions can be found and the $\mathrm{BH}$ ceases to be a $\mathrm{BH}$ with a naked singularity.

\section{Throne-Morris Wormhole Solutions}


Here we will follow the approach used in Ref. [13]. In the radial coordinate system, if the radius is $r$ then the circumference can be represented by $2 \pi r$, where $\ell= \pm$ shows the WH mouth distance from throat with $\ell+$ in one location and $\ell-$ in the other faraway location. Two independent factor defines the WH solutions through $r$ - where $b(r)$ the shape function defined by $\frac{d \ell}{d r}= \pm(1-b / r)^{-1 / 2}$ and $\Phi(r)$ the redshift function defined by $g_{u}=-e^{2 \Phi}$. The related metric can be given by,

$d s^{2}=-e^{2 \Phi} c^{2} d t^{2}+\left(1-\frac{b}{r}\right)^{-1} d r^{2}+r^{2}\left(d \theta^{2}+\sin ^{2} \theta d \phi^{2}\right)$

Static observers will be referenced by the orthonormal basis of the reference frame as: $e_{\hat{t}}=e^{-\Phi} e_{t}, e_{\hat{r}}=$ $\left(1-\frac{b}{r}\right)^{1 / 2} e_{r}, e_{\widehat{\theta}}=r^{-1} e_{\theta} e_{\widehat{\Phi}}=(r \sin \theta)^{-1} e_{\Phi}$

The throat is minimum at $r$ (we have denoted $r=0$ in section 5 for the computation of the temporal intervals which is just a value to represent and doesn't mean the radius has been collapsed) where $r=b=b_{0}$ throughout the spacetime $1-b / r \geq 0$ as $\ell \rightarrow \pm \infty$ (the flaring out conditions for the asymptotically flat regions of the WHs location) such that $b / r \rightarrow 0$ so $r \cong|\ell|$.

Static observers will measure the radial velocity given as: $v(r) ; \gamma \equiv\left(1-\left(\frac{v}{c}\right)^{2}\right)^{-1 / 2}$

Trip begins at $\ell=-\ell_{1}$, ends at $\ell=+\ell_{2} ; v=0$ at $-\ell_{1}$ and $+\ell_{2}$; gravity is weak at $-\ell_{1}$ and $+\ell_{2}: \frac{b}{r} \ll 1$, $|\Phi| \ll 1,\left|\Phi^{\prime c^{2}}\right| \lesssim g_{\oplus .}=$ Earth Gravity. Here and below ${ }^{\prime} \equiv \frac{d}{d r}=\left(1-\frac{b}{r}\right)^{-\frac{1}{2}} d / d \ell$

As singularity parameter $\Phi$ is finite everywhere, so it denotes a singularity free tunnel, however we will used a method to smoothen out the singularity by tunneling the affine metric in sections 6 and $7, t$ measures the proper time in asymptotically flat regions $\Phi \rightarrow 0, \ell \rightarrow \pm \infty$.

The whole trip will take a value as: $\Delta \tau=\int_{-\ell_{1}}^{+\ell_{2}}(v \gamma)^{-1} d \ell \lesssim 1 y r$. and also as seen by static observers at $-\ell_{1}$ and $+\ell_{2} \Delta t=\int_{-\ell_{1}}^{+\ell_{2}}\left(v e^{\Phi}\right)^{-1} d \ell \lesssim 1 y r$

Acceleration felt by the traveler $\left|\frac{e^{-\Phi} d\left(\gamma e^{\Phi}\right)}{d \ell}\right| \lesssim \frac{g_{\oplus}}{c^{2}}=\frac{1}{0.97} 1 y r$

Traveler feels tidal acceleration between different parts of the body with magnitude $\lesssim g_{\oplus}$ :

$\left|(1-b / r)\left[-\Phi^{\prime \prime}+\frac{1}{2} \frac{\Phi^{\prime}}{\left(b^{\prime}-\frac{b}{r}\right)(r-b)}-\left(\Phi^{\prime}\right)^{2}\right]\right| \lesssim\left(1 / 10^{10} c m\right)^{2}$

$\left|\frac{1}{2}\left(\frac{\gamma}{r}\right)^{2}\left[\left(\frac{v}{c}\right)^{2}\left(b^{\prime}-\frac{b}{r}\right)+2(r-b) \Phi^{\prime}\right]\right| \lesssim\left(1 / 10^{10} c m\right)^{2}$

Stress-energy tensor measured by static observers as,

$T_{\hat{t} \hat{t}}=\rho c^{2}=($ mass energy density $)$

$T_{\hat{r} \hat{r}}=-\zeta=-($ radial tension $)$ 
$T_{\widehat{\theta} \widehat{\theta}}=T_{\widehat{\phi} \hat{\phi}}=p=($ lateral pressue $)$

(15)

Unstable because $\zeta \gg \rho c^{2}$ and that demands exotic matter where $\rho=b^{\prime} /\left[8 \pi G c^{-2} r^{2}\right], \zeta=\left[\frac{b}{r}-\right.$ $\left.2(r-b) \Phi^{\prime}\right] /\left[8 \pi G c^{-4} r^{2}\right], p=\left(\frac{r}{2}\right)\left[\left(\rho c^{2}-\zeta\right) \Phi^{\prime}-\zeta^{\prime}\right]-\zeta$

Einstein field equations can be given as: With ${ }^{\prime} \equiv \frac{d}{d r}=\left(1-\frac{b}{r}\right)^{\frac{1}{2}} d / d \ell$

$\rho=\frac{b^{\prime}}{8 \pi G c^{-2} r^{2}}$

$\zeta=\frac{\frac{b}{r}-2(r-b) \Phi \prime}{8 \pi G c^{-4} r^{2}}$

$p=\frac{r}{2}\left[\left(\rho c^{2}-\zeta\right) \Phi^{\prime}-\zeta^{\prime}\right]-\zeta$

(16)

At the throat $r=b=b_{0}, \zeta$ and $p$ depend on throat shape.

\section{IV. $f(R, T)$ Solutions of Throne-Morris Wormhole}

Assuming the redshift function is constant $(\phi \rightarrow 0)$, in the modified gravitational theory $f(R, T)=R+$ $\alpha R^{2}+<T$, with $\alpha>0$ in the modified gravity for non-exotic matter, the wormhole metric can be written as [20],

$\frac{b^{\prime}}{r^{2}}=\frac{\left[\left(8 \pi+\frac{3 \varkappa}{2}\right) \rho-\frac{\alpha\left(p_{r}+2 p_{t}\right)}{6}-\frac{2 \alpha b^{\prime 2}}{r^{4}}\right]}{2 \alpha R+1}=\rho_{\mathrm{eff}} \equiv T_{\mu \nu}^{\mathrm{eff}}$

$\frac{b}{r^{3}}=\frac{\left[-\left(8 \pi+\frac{7 \varkappa}{6}\right) p_{r}+\frac{\varkappa}{2}\left(\rho-\frac{2 p_{t}}{3}\right)-\frac{2 \alpha b^{\prime 2}}{r^{4}}\right]}{2 \alpha R+1}=p_{r_{\mathrm{eff}}} \equiv T_{\mu \nu}^{\mathrm{eff}}$

$\frac{b^{\prime} r-b}{2 r^{3}}=\frac{\left[-\left(8 \pi+\frac{4 \varkappa}{3}\right) p_{t}+\frac{\varkappa}{2}\left(\rho-\frac{2 p_{r}}{3}\right)-\frac{2 \alpha b^{\prime 2}}{r^{4}}\right]}{2 \alpha R+1}=p_{t_{\mathrm{eff}}} \equiv T_{\mu \nu}^{\mathrm{eff}}$

(17)

The Ricci Scalar for the WH metric is,

$R=\frac{2 b^{\prime}}{r^{2}}$

(18)

For shape function $b(r)=r_{0}^{2} \frac{e^{r_{0}-r}}{r}$, throat is at $r_{0}=1$ [20],

$\rho=\frac{e^{-2 r+r_{0}}(1+r) r_{0}^{2}\left(-2 e^{r} r^{4}(6 \pi+\curlywedge)+e^{r_{0}}(1+r) r_{0}^{2} \alpha(72 \pi+11 \curlywedge)\right)}{3 r^{8}\left(32 \pi^{2}+12 \pi \curlywedge+\curlywedge^{2}\right)}$

$p_{r}=\frac{e^{-2 r+r_{0}} r_{0}^{2}\left(e^{r} r^{4}(12 \pi+(4+r) \curlywedge)+e^{r_{0}}(1+r) r_{0}^{2} \alpha(24 \pi(-1+r)-(13+r) \curlywedge)\right.}{3 r^{8}\left(32 \pi^{2}+12 \pi \curlywedge+\iota^{2}\right)}$ 
[1] DEC: $\rho \geq\left|p_{i}\right|$

[2] WEC: $\rho \geq 0 ; \rho+p_{i}>0$

[3] NEC: $\rho+p_{i} \geq 0$

[4] SEC: $\rho+p_{r}+2 p_{t} \geq 0$

Where $i=r, t$

The WH models considered here are asymptotically flat as for $r \rightarrow \infty,\left(1-\frac{b(r)}{r}\right) \rightarrow$. For this hybrid functions, the energy density $\rho$ of the throat is positive when (i) for positive $\alpha>\left(\frac{2(\curlywedge+6 \pi)}{11 \curlywedge+72 \pi}\right)$, (ii) $\alpha>\frac{1}{6}$ with $\curlywedge=0$, (iii) Both $\curlywedge$ and $\alpha$ are negative satisfying the inequalities $|\alpha|<\left(\frac{2(|<|-6 \pi)}{72 \pi-11|<|}\right)$ with $6 \pi<|<|<\frac{72 \pi}{11}$, (iv) $\alpha$ is positive satisfying the inequalities $\alpha>\left(\frac{2(6 \pi-|<|)}{72 \pi-11|<|}\right)$ with $\frac{72 \pi}{11|<|}<|<|<6 \pi$.

The choice of shape function is very important as it gives the following results [33]:

- For negative values of $\curlywedge$ NEC for radial pressure is obeyed but, NEC for transverse pressure and DEC for radial pressure are not obeyed.

- For positive values of $<$ all energy conditions are violated.

- For the limit $\longleftrightarrow 0$, WHs are obtained with normal matter in $f(R)$ model and the energy conditions are valid in case of $f(R)=R+\alpha R^{n}$ with $\alpha \geq 0, n=2$.

When I considered $n=2$ then different properties of WHs are found obeying and disobeying the energy conditions which is different from normal (Einstein) WHs where energy conditions are only violated.

\section{V. $\quad f(R, T)$ Solutions of Temporal Intervals In Throne-Morris Wormhole}

The effective stress-energy tensor can be replaced by [20],

$R_{\mu \nu}-\frac{1}{2} g_{\mu \nu} R=T_{\mu \nu}^{\mathrm{eff}} \equiv \frac{1}{f_{R}(R, T)}\left(\left[\left(8 \pi+f_{T}(R, T) T_{\mu \nu}+P g_{\mu \nu} f_{R}(R, T)\right]+\left[\frac{1}{2}\left[f(R, T)-R f_{T}(R, T] g_{\mu \nu}\right]-\right.\right.\right.$ $\left.\left[\left(g_{\mu \nu} \square-\nabla_{\mu} \nabla_{\nu}\right) f_{R}(R, T)\right]\right)$

The Kerr metric can be re-written in the below form from equation (1) [34],

$c^{2} d \tau^{2}=\left(g_{t t}-\frac{g_{t \phi}^{2}}{g_{\phi \phi}}\right) d t^{2}+g_{r r} d r^{2}+g_{\theta \theta} d \theta^{2}+g_{\phi \phi}\left(d \phi+\frac{g_{t \phi}}{g_{\phi \phi}} d t\right)^{2}$

The metric perturbation can be given for a colatitude $\theta$ which obeys a corotating reference frame having angular speed $\Omega_{k}$ depending on the radial distance $r$ and the mass $M$ where the equation can be derived with respect to an inertial frame from the perspectives of a rotating central mass to take part on the latter's frame by, 
$\Omega_{k}=-\frac{g_{t \phi}}{g_{\phi \phi}}=\frac{r_{s} a r c}{\rho^{2}\left(r^{2}+\alpha^{2}\right)+r_{s} a^{2} r \sin ^{2} \theta}=\left.\frac{r_{s} a c}{r^{3} a^{2} r+r_{s} \alpha^{2}}\right|_{\text {in the equatorial plane }}$

$M=\left.\int T^{00} d^{3} x\right|_{\text {mass of the rotating body }}$

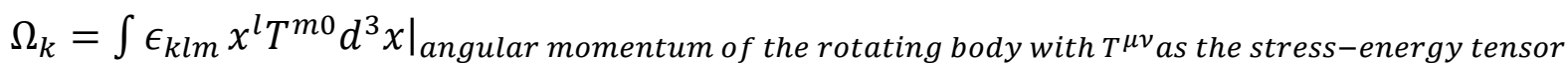

$\xi=\int_{\frac{+\ell-\ell}{2}}^{ \pm \ell} m^{d-4} \wedge n^{d-4} \wedge o^{d-4} \wedge p^{d-4} \ldots \ldots \ldots \ldots\langle b(r), \Phi(r)\rangle$

Where $m, n, o, p \ldots$ are tangent to hyperplane $\mathcal{H}$ in the spatial sheaf of $d-4$ where the infinite multiplicity of the hyperplane is given by,

$\xi\left(* \mathcal{S}^{2 D}\right) \equiv \prod_{i=1}^{ \pm \infty} H_{i}$

With $* \mathcal{S}^{2 D}$ satisfying the many order temporal permutations with each event $*$ engaged with the $2 D$ temporal foliations in the order of,

$* \mathcal{S}^{2 D} \equiv \prod_{*=1}^{ \pm \infty} * S$

Giving us the relation of the foliated events $\mathcal{F}^{d \sigma^{2}}$ with $d \sigma^{2}=d x^{2}+d y^{2}+d z^{2}=d r^{2}+r^{2} d \theta^{2}+$ $r^{2} \sin ^{2} \theta d \varphi^{2}$ given as,

$\mathcal{F}^{d \sigma^{2}}=\prod_{i=1}^{ \pm \infty} \prod_{*=1}^{ \pm \infty} H_{i} \otimes * S$

This is ofcourse a one way $d-4$ spatial and $2 D$ temporal sheath because the tangent bundles $\xi$ has a restricted vector $\vec{e}_{1}, \vec{e}_{2}, \vec{e}_{3} \ldots$ that is projecting holographically in the interval $\left[\frac{+\ell-\ell}{2}, \pm \ell\right]$ as a projective function $\mathcal{P}$ and $\tilde{\mathcal{P}}$ to $\mathcal{F}^{d \sigma^{2}}$ given as,

$\mathcal{P}\left[\frac{+\ell-\ell}{2}, \pm \ell\right] \overrightarrow{(P A S T)} \mathcal{F}^{d \sigma^{2}}$

$\tilde{\mathcal{P}}\left[\frac{+\ell-\ell}{2}, \pm \ell\right] \overrightarrow{(F U T U R E)} \overrightarrow{\mathcal{F}} d \sigma^{2}$

(26)

With a one way projection vector basis $\vec{e}_{1}, \vec{e}_{2}, \vec{e}_{3} \ldots$ going to,

$\vec{e}_{1} \mathcal{P}, \vec{e}_{2} \mathcal{P}, \vec{e}_{3} \mathcal{P}$... over the $d-4$ sheath in $\left.\mathcal{H}\right\rfloor_{\text {to the sheath from the outside }}$

$\vec{e}_{1} \tilde{\mathcal{P}}, \vec{e}_{2} \tilde{\mathcal{P}}, \vec{e}_{3} \tilde{\mathcal{P}}$... over the $d-4$ sheath in $\left.\mathcal{H}\right|_{\text {to the sheath from the outside }}$

$e\left(t_{c}\right)=2 \pi\left[\frac{t_{1}-t_{0}}{\tau_{c}}\right]^{\vec{e}_{1} \mathcal{P}+\vec{e}_{2} \mathcal{P}+\vec{e}_{3} \mathcal{P} \ldots \vec{e}_{1} \tilde{\mathcal{P}}+\vec{e}_{2} \tilde{\mathcal{P}}+\vec{e}_{3} \tilde{\mathcal{P}} \ldots}$

Where $e\left(t_{c}\right)$ is the exponent functions of the $t_{c}$ time loop where past intersects future and again past through a phase rotation of $2 \pi$ having the period $\tau_{c}$ with the origin $t_{0}$ to the time $t_{1}$. However, approaching from the 
period increases or decreases by virtue of a temporal dilation factor $\tau_{c}=t \sqrt{1-\frac{V^{2}}{c^{2}}}$ where in the WH journey $V^{2} \approx c^{2}$ and thus $\tau_{c}=0$, which when introduced in the equation

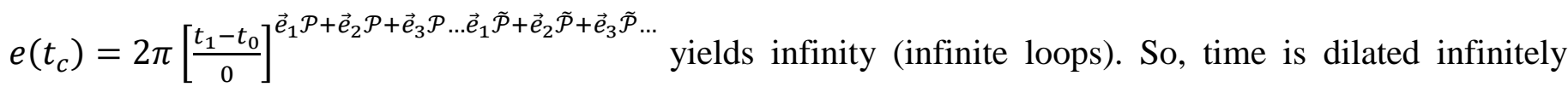
inside the WH. However, we can give the parameters by denoting ' + ' as the elongation of time when its more dilated and ' - ' as the contraction of time when its less dilated approaching to the normal time $\tau_{c}=t$, while where the time won't change can be denoted as $\mathcal{O}$ the relations are,

\begin{tabular}{|c|c|c|c|c|c|c|c|c|c|}
\hline $\begin{array}{c}\text { No. } \\
1 .\end{array}$ & $\begin{array}{c}\text { INTERVAL } \\
-\ell\end{array}$ & $\begin{array}{c}1^{s t} R A D I U S \\
(r=-\infty)\end{array}$ & $\underset{m \rightarrow}{M A P .}$ & $\begin{array}{c}2^{\text {nd }} \text { RADIUS } \\
(r=+\infty)\end{array}$ & $\begin{array}{l}M A P . \\
\Rightarrow\end{array}$ & $\begin{array}{c}\text { INTERVAL } \\
+\ell\end{array}$ & $\stackrel{M A P .}{\longrightarrow}$ & $\begin{array}{c}\text { POLES } \\
t,-,+,-, t\end{array}$ & $\begin{array}{c}\text { FUNCTION } \\
f(t)\end{array}$ \\
\hline 2. & $+\ell$ & $(r=+\infty)$ & $m \rightarrow$ & $(r=-\infty)$ & $\Longrightarrow$ & $-\ell$ & $\longrightarrow$ & $t,-,+,-, t$ & $f(t)$ \\
\hline 3. & $\frac{+\ell-\ell}{2}$ & $(r=0)$ & $m \rightarrow$ & $(r=+\infty)$ & $\Longrightarrow$ & $+\ell$ & $\longrightarrow$ &,,$+- t$ & $f(t)$ \\
\hline 4. & $+\ell$ & $(r=+\infty)$ & $m \rightarrow$ & $(r=0)$ & $\Longrightarrow$ & $\frac{+\ell-\ell}{2}$ & $\rightarrow$ & $t,-,+$ & $f(t)$ \\
\hline 5. & $\frac{+\ell-\ell}{2}$ & $(r=0)$ & $m \rightarrow$ & $(r=-\infty)$ & $\Longrightarrow$ & $-\ell$ & $\longrightarrow$ &,,$+- t$ & $f(t)$ \\
\hline 6. & $-\ell$ & $(r=-\infty)$ & $m \rightarrow$ & $(r=0)$ & $\Longrightarrow$ & $\frac{+\ell-\ell}{2}$ & $\longrightarrow$ & $t,-,+$ & $f(t)$ \\
\hline 7. & $\frac{+\ell-\ell}{2}$ & $(r=0)$ & $m \rightarrow$ & $(r=0)$ & $\Rightarrow$ & $\frac{+\ell-\ell}{2}$ & $\rightarrow$ & $\mathcal{O}$ & $\times$ \\
\hline 8. & $-\ell$ & $(r=-\infty)$ & $m$ & $(r=-\infty)$ & $\Longrightarrow$ & $-\ell$ & $\rightarrow$ & $\mathcal{O}$ & $x$ \\
\hline 9. & $+\ell$ & $(r=+\infty)$ & $m \rightarrow$ & $(r=+\infty)$ & $\Rightarrow$ & $+\ell$ & $\longrightarrow$ & $\mathcal{O}$ & $x$ \\
\hline
\end{tabular}

Table 1. Computation of spacelike intervals where at the throat, the radius is assumed to be zero, however, in reality it is not, the throat radius has a finite value but for the simplicity of the calculation, we have taken $r=0$.

The interesting parts are the last 3 parts (e.g. 7, 8,9) where,

- 7 stands for a collapsing wormhole when the traveler is at the middle,

- 8 stands for the traveler wandering asymptotically at the mouth of the WH in his present universe.

- 9 stands for the traveler wandering asymptotically at the mouth of the WH at his destination universe (which will collapsed him to die as we see later)

$$
\begin{array}{ccc}
\text { Terms } & \alpha>0 & \alpha<0 \\
\rho & \geq 0 \text { for } r \in(0, \infty) & \leq 0 \text { for } r \in(0, \infty) \\
\rho+\rho_{r} & \geq 0 \text { for } r \in(0, \infty) & \leq 0 \text { for } r \in(0, \infty) \\
\rho+\rho_{t} & \geq 0 \text { for } r \in(0, \infty) & \leq 0 \text { for } r \in(0, \infty) \\
\rho-\left|p_{r}\right| & \geq 0 \text { for } r \in(0, \infty) & \leq 0 \text { for } r \in(0, \infty) \\
\rho-\left|p_{t}\right| & \geq 0 \text { for } r \in(0, \infty) & \leq 0 \text { for } r \in(0, \infty) \\
\rho+p_{r}+2 p_{t} & \leq 0 \text { for } r \in(0, \infty) & \geq 0 \text { for } r \in(0, \infty)
\end{array}
$$

Table 2. Summary of results for $b(r)=r_{0}^{2} \frac{e^{r_{0}-r}}{r}$ with $\curlywedge=1$ and different $\alpha[20,35]$. 


$$
\begin{array}{cccc}
\text { Terms } & \wedge=1 & \wedge=76 & \wedge=-13 \\
\rho & \geq 0 \text { for } r \in(0, \infty) & \geq 0 \text { for } r \in(0, \infty) & \leq 0 \text { for } r \in(0, \infty) \\
\rho+\rho_{r} & \geq 0 \text { for } r \in(0, \infty) & \geq 0 \text { for } r \in(0, \infty) & \geq 0 \text { for } r \in(0, \infty) \\
\rho+\rho_{t} & \geq 0 \text { for } r \in(0, \infty) & \geq 0 \text { for } r \in(0, \infty) & \text { violated for a range of } r \\
\rho-\left|p_{r}\right| & \geq 0 \text { for } r \in(0, \infty) & \text { violated for small } r & \leq 0 \text { for } r \in(0, \infty) \\
\rho-\left|p_{t}\right| & \geq 0 \text { for } r \in(0, \infty) & \geq 0 \text { for } r \in(0, \infty) & \leq 0 \text { for } r \in(0, \infty) \\
\rho+p_{r}+2 p_{t} & \leq 0 \text { for } r \in(0, \infty) & \leq 0 \text { for } r \in(0, \infty) & \geq 0 \text { for } r \in(0, \infty)
\end{array}
$$

Table 3. Summary of results for $b(r)=r_{0}^{2} \frac{e^{r_{0}-r}}{r}$ with $\alpha=1$ and different $<[20,35]$.

$\begin{array}{cccc}\text { Coordinates: } & X & Y & Z \\ \text { NO. } & \text { START DOMAIN } & \text { END DOMAIN } & \text { POLES } \\ 1 . & -1 & +1 & .5^{1} \\ 2 . & +1 & -1 & .5^{2} \\ 3 . & 0 & +1 & -.3^{1} \\ 4 . & +1 & 0 & .3^{2} \\ 5 . & 0 & -1 & -.3^{3} \\ 6 . & -1 & 0 & .3^{4} \\ 7 . & 0 & 0 & .0^{1} \\ 8 . & -1 & -1 & .0^{2} \\ 9 . & +1 & +1 & .0^{3}\end{array}$

Table 4. Data points to be denoted by the graph where $-1=-\infty,+1=+\infty, 0=0$ in the $1^{\text {st }}$ radius (start domain in $\mathrm{X}$ axis), $2^{\text {nd }}$ radius (end domain in the $\mathrm{Y}$ axis), poles (where each interval to be counted after decimal for the convenience of plotting with power indices as a symbol of consecutive repetition) as depicted in table 1 .

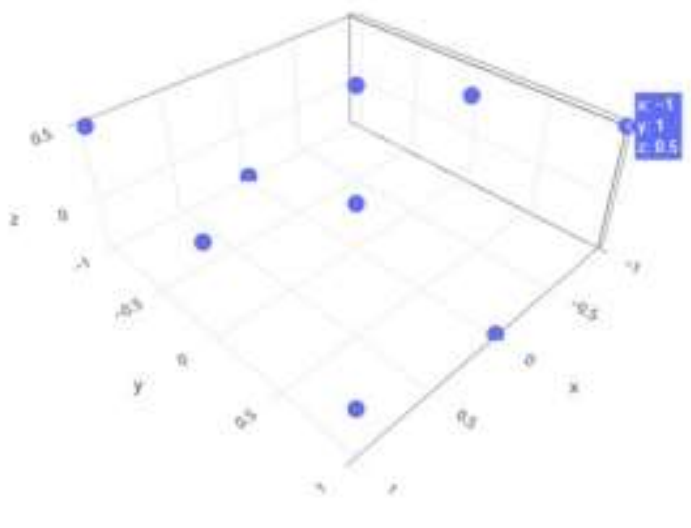

Figure 3. Pole No. 1 Plocted in the landecape

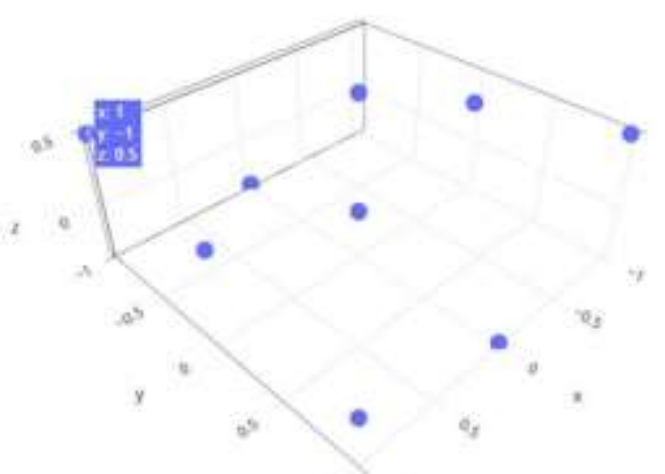

Figure 4. Fole No. 2 Plotted in the lansacape. 


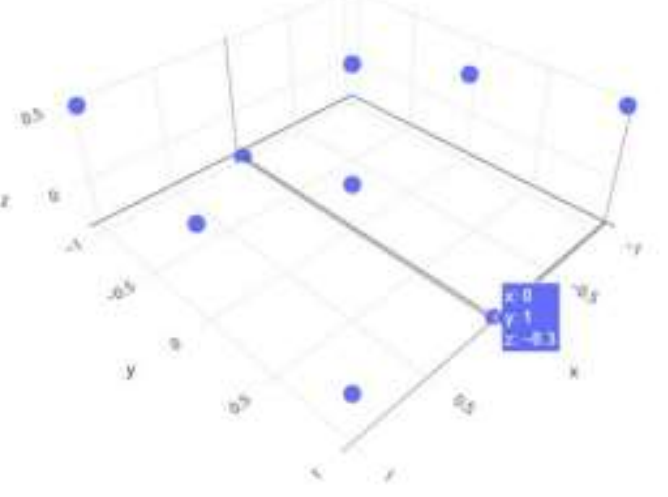

Figure 5. Pole No, 3 Plorted plotred in the landecape.

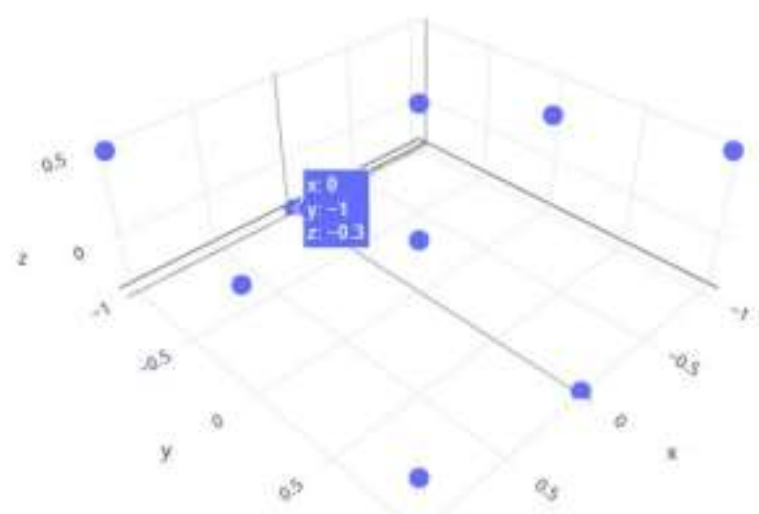

Figure 7. Pole No. 5 plestes in the lanthespe

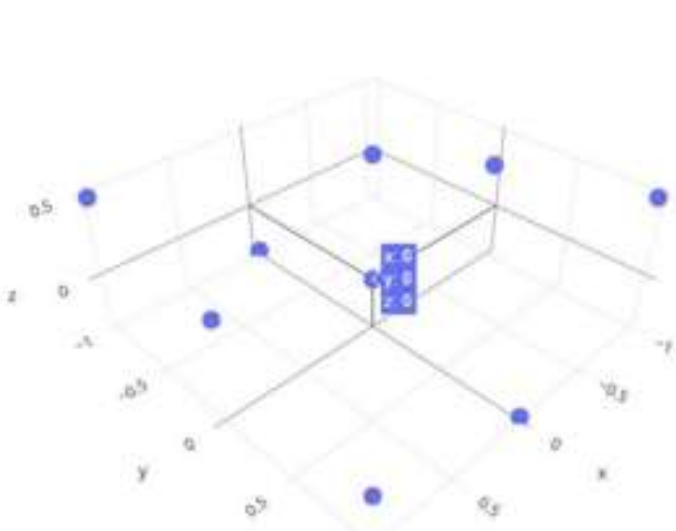

Figure 9. Pole No. 7 Plotted in the iandocabe.

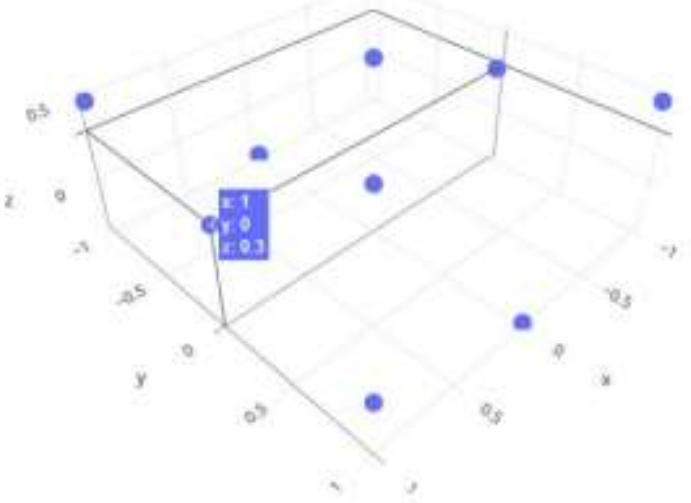

Figore 6. Pole No. 4 Plettrod in the lenscapt

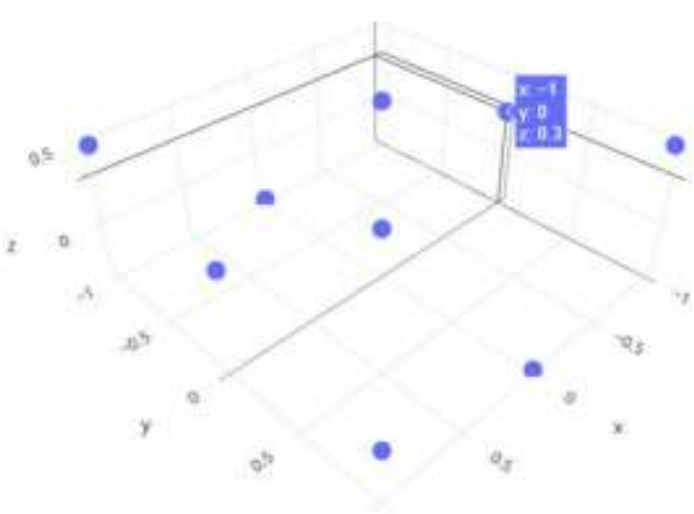

Figure 8. Pole No. 8 Plottes in the landocape

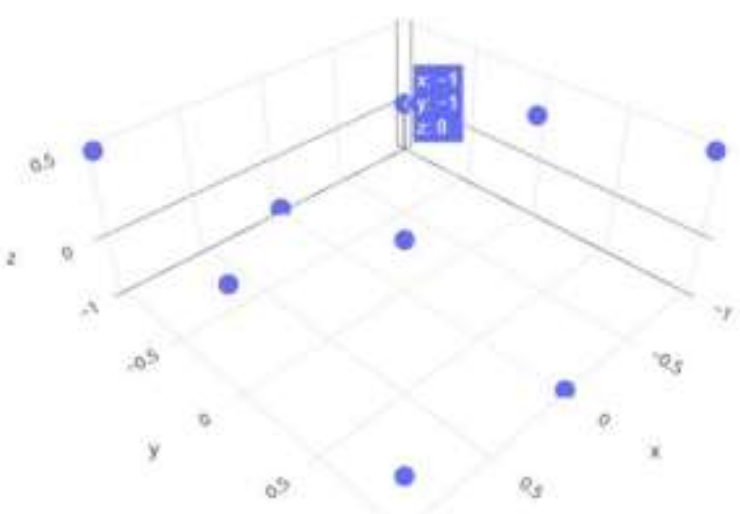

Figure 10. Pole hio. s Plotted in the landucape 


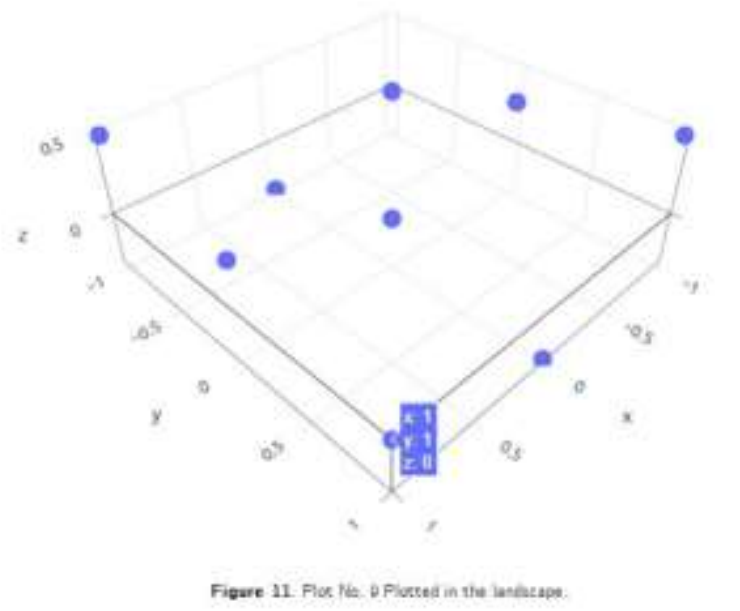

$Z$ coordinates indicate 9 poles as such shown in the figures with the respective values of $.5^{1}, .5^{2}, .3^{1}, .2^{1}, .3^{2}, .2^{2}, .0^{1}, .0^{2}, .0^{3}$. Here, superscripts of $1,2,3$ are just labeling parameters used to denote the unique orientations of poles while the numbers after dots (.) denotes the amount of "phase times" that has been passed with each crossing intervals. To denote the boundary conditions of the "hologram" consisting of different projections of 'poles' which will act here as 'timelines' we need to establish a $d-4$ sheaf boundary over the hypersurface $\epsilon^{i} \partial \mathcal{H}^{d-4}$ over each combinations denoted by a unique value of $i$. The results will apply in two form ways,

- Interconnected (or intersecting) timeline combinations having 9 poles as $9 \times 9$ ! as 3265920 .

- Unique timeline combinations involving $.5^{j}, .0^{j}, .3^{j},-.3^{j}$ in 4 -orders among the 9 poles as $.5^{1}, .5^{2},-.3^{1}, .3^{2},-.3^{3}, .3^{4}, .0^{1}, .0^{2}, .0^{3}$ with $j=1,2,3,4$ and if we will compute with this as because the traveler can only able to visualize properly the grids resulting in $4 \times 4$ ! i.e., 96 unique combinations of time lapse provided below.

$$
\left.\begin{array}{ccccc}
1^{\text {st }} \text { term } & .5^{j} & .3^{j} & -.3^{j} & .0^{j} \\
2^{\text {nd }} \text { term } & .3^{j} & -.3^{j} & .0^{j} & .5^{j} \\
3^{\text {rd }} \text { term } & -.3^{j} & .0^{j} & .5^{j} & .3^{j} \\
4^{\text {th }} \text { term } & .0^{j} & -.3^{j} & .3^{j} & .5^{j} \\
\vdots & \ddots & \ddots & \ddots & \ddots \\
\vdots & \ddots & \ddots & \ddots & \ddots \\
96^{\text {th }} \text { term } & .3^{j} & .0^{j} & .5^{j} & -.3^{j}
\end{array}\right\}\left(\epsilon^{i} \partial \mathcal{H}^{d-4}\right) \rightrightarrows * \mathcal{S}^{2 D}
$$

- This provides us the scattered time lapse grid (where each number denotes a singular timelike grid projecting inwards where the person inside the WH can see the outside as part of a hazy/random hologram which we actually mean by scattered as opposite to unique where he/she can see 96 clear/unique time lapse/timelines with spacelike metric) as $3265920-96=3265824$ as the temporal mesh over simulating surface given by $\mathcal{M}^{i} \otimes * \mathcal{S}^{2 D}$. Having the boundary $-\left(\epsilon^{i} \partial \mathcal{H}^{d-4}\right)$.

Therefore, the $d-4$ sheath boundary can be represented as

$$
\begin{aligned}
& \sum_{\substack{i=1 \\
\text { in }}}^{96} \sum_{\substack{i=1 \\
\text { in }}}^{3265824}\left(\epsilon^{i} \partial \mathcal{H}^{d-4}\right)-\left(\epsilon^{i} \partial \mathcal{H}^{d-4}\right) \equiv\left(\epsilon^{96} \partial \mathcal{H}^{d-4}\right)-\left(\epsilon^{3265824} \partial \mathcal{H}^{d-4}\right) \equiv \\
& \left(\epsilon^{i} \partial \mathcal{H}^{d-2}\right)-\left(\epsilon^{i} \partial \mathcal{H}^{d-2}\right) \\
& -\left(\epsilon^{3265728} \partial \mathcal{H}^{d-4}\right)
\end{aligned}
$$

So, we are getting a total of [3265728] $d-4$ sheath boundary as $-\left(\epsilon^{3265728} \partial \mathcal{H}^{d-4}\right)$. The $(-)$ sign represents it as an anti-boundary with the timelines projecting from the outside world and penetrating inside the 
WH as a hologram. (-) sign also indicates a one-way sheath. This represents a one way superficially hypermembrane where the time traveler is totally covered with and can see the scattered and unique timelines in a spacelike frame, however, the persons he/she is watching is totally ignorant of the time traveler or person inside the WH observing it thereby there will no penetration of the present timeline with the past/future, thus avoiding all sorts of paradoxes.

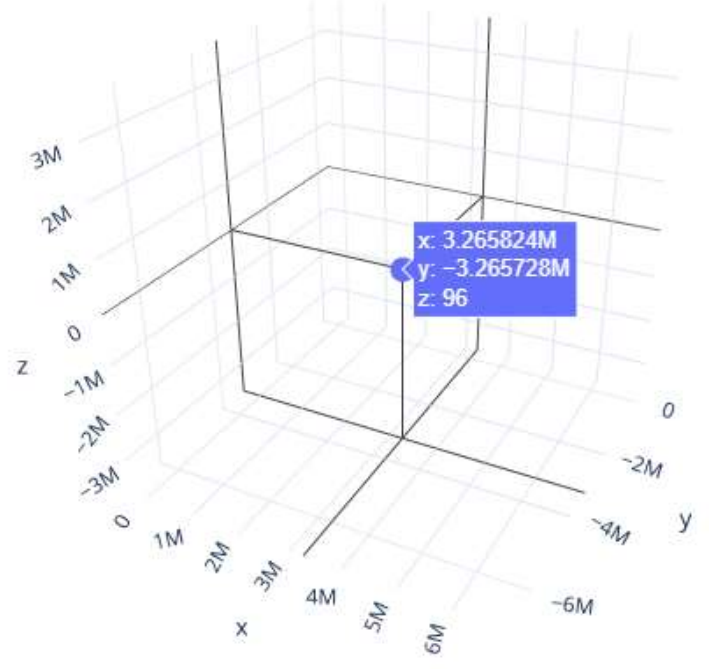

Figure 12. Plotted in the $Z$ axis 'unique pole' $96, X$ axis 'scattered pole' 3265824 and this intersection shows the $d-4$ sheath boundary of -3265728 intersecting ' 1 ' (Y axis) of it to the junction $(X, Y, Z)$ of the $96^{\text {th }}$ unique pole to $3265824^{\text {th }}$ scattered loops representing the region $\frac{+\ell-\ell}{2}$.

Now, we will obtain the total space-time dimensions excluding the second dimensions of time through Lorentz-Generators as [36, 37],

$\left[A^{-i}, A^{-j}\right]=-\frac{1}{\left(p^{+}\right)^{2}} \sum_{n=1}^{\infty}\left(\alpha_{-n}^{i} \alpha_{-n}^{j}-\alpha_{-n}^{j} \alpha_{-n}^{i}\right)(\Delta n-n)$

$\Delta n=n\left(\frac{D-2}{8}\right)+\frac{1}{n}\left(2 \alpha_{N S}-\frac{D-2}{8}\right)$

For Lorentz invariance $\left[A^{-i}, A^{-j}\right]=0$. This will only happen when $n\left(\frac{D-2}{8}\right)$ collapses to $n$ and when it is equal to 1 , and $\frac{1}{n}\left(2 \alpha_{N S}-\frac{D-2}{8}\right)$ becomes 0 .

When,

$\left(2 \alpha_{N S}-\frac{D-2}{8}\right)=0$

Then,

$n\left(\frac{D-2}{8}\right)=1$

(31)

Or, 
And when $\left(2 \alpha_{N S}-\frac{D-2}{8}\right)=0$ and $D=10$, then $\alpha_{N S}=\frac{1}{2}$ which is the normal ordering constant of $N S-$ sector in superstring theory.

Then, $D-($ normal space - time dimensions $)=d-4$ sheath.

\section{Tunneling Kerr's Ring Singularity}

As it's the affine structure of the WH to be free from singularity, so we will try to shield the ring singularity of the Kerr spacetime with blown event horizon by an approach of tunneling or getting through it, just like inserting a higher dimensional hollow pipe in between the ring circumference so that no perturbations can occur in the time travelers frame while he is travelling through it, where in the next section, i.e., section 7 , we will show that those present moments are actually a complex moments, rather a complex conjugate with the time being imaginary to smoothen out the singularity [47]. So, let us focus on the interval between two nearby regions around the middle (or throat) of the WH as,

$\left.\frac{+\ell-\ell}{2+\delta}\right|_{(\mathrm{Up})}<\frac{+\ell-\ell}{2}>\left.\frac{+\ell-\ell}{2+\delta}\right|_{(\mathrm{down})} \overrightarrow{\text { lmplles }}\left[\left.\frac{+\ell-\ell}{2+\delta}\right|_{(\mathrm{Up})},\left.\frac{+\ell-\ell}{2+\delta}\right|_{(\mathrm{down})}\right]$

Where $2+\delta$ being a small extension from 2 . The shape is a hypercylinder or spherinder with a $3 D$ hypertube connected two surface of two 2 - spheres at both ends each. And this has the embed $2 D$ Kerr ring and $2 D$ time being embedded as a circular loop. Cauchy horizon lies further away at the end of the hypertube where there is the location of $d-4$ spatial sheath.

The metric can be given by spacelike hypersurfaces with $2 D$ time and $2 D$ ring singularity (both embedded) as,

$\left.d l^{2}\right]_{\left[\left.\frac{+\ell-\ell}{2+\delta}\right|_{(\mathrm{Up})},\left.\frac{+\ell-\ell}{2+\delta}\right|_{(\mathrm{down})}\right]}=-d t_{1}^{2}-d t_{2}^{2}+d r^{2}+d \varphi^{2}+d \theta^{2}+d \omega^{2}+d r_{1}^{2}+r^{2} d \theta_{1}^{2}$

Given [26,38],

$-d t_{1}^{2}-d t_{2}^{2}=2 D$ temporal dimensions

$d r^{2}=d\left(\sqrt{x^{2}+y^{2}+z^{2}}\right)^{2}$

$d \varphi^{2}=d\left(\arctan \frac{y}{x}\right)^{2}$

$d \theta^{2}=d\left(\operatorname{arccot} \frac{z}{\sqrt{x^{2}+y^{2}}}\right)^{2}$

$d \omega^{2}$

$d r_{1}^{2}+r^{2} d \theta_{1}^{2}=$ metric of the hollow ring

(35)

The occupied volume over which the $d-4$ spatial sheath takes place is given by excluding the middle throat interval to make the region on the (Up) and (down) free from singularity is defined by the interval, 
$\left[\left(\left.\frac{+\ell-\ell}{2+\delta}\right|_{(\mathrm{Up})}-\frac{+\ell-\ell}{2}\right),\left(\left.\frac{+\ell-\ell}{2+\delta}\right|_{(\mathrm{down})}-\frac{+\ell-\ell}{2}\right)\right]_{\text {assuming Kerr ring lies in the middle region } \frac{+\ell-\ell}{2}}$

The hypervolume interval domain is given by,

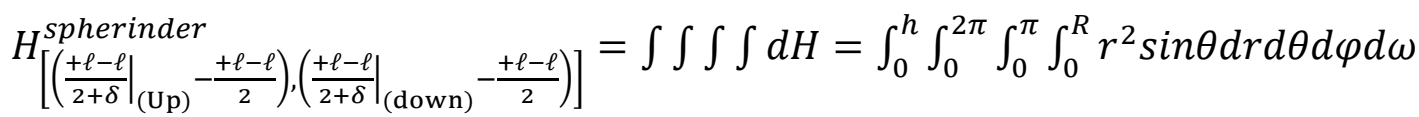

This volume will be on a limited interval surrounding the singularity, and we will see that how this area represents complex/complex conjugate time through it from the perspective of the time travelers frame.

\section{Time Trajectories}

The time trajectories of the interval has been represented by means of the tetrad formulations which shows the different parameters of the trajectories as,

$\left.\begin{array}{cccc}\text { Past } & \mathcal{P}^{m} & {[ \pm \ell, \pm \ell]} & \mathcal{P}^{m} \mathcal{P}_{m}=0 \\ \text { Future } & \tilde{\mathcal{P}}^{m} & {[ \pm \ell, \pm \ell]} & \tilde{\mathcal{P}}^{m} \tilde{\mathcal{P}}_{m}=0 \\ \text { Present } & \tilde{\mathcal{P}}^{m}+i \mathcal{P}^{m} & {[ \pm \ell, \pm \ell]} & \mathcal{P}^{m} \tilde{\mathcal{P}}_{m}=-1 \\ \text { Present conjugate } & \tilde{\mathcal{P}}^{m}-i \mathcal{P}^{m} & {\left[\left.\frac{+\ell-\ell}{2+\delta}\right|_{(\text {Up) }},\left.\frac{+\ell-\ell}{2+\delta}\right|_{(\text {down })}\right.}\end{array}\right] \begin{gathered}\tilde{\mathcal{P}}^{m} \mathcal{P}_{m}=-1 \\ \end{gathered}$

Table. 5 Computation of the values for the construction of the NP-tetrad [39, 40, 41, 42, 43, 44, 45].

The tetrad can be represented by: $\left\{\mathcal{P}^{m}, \widetilde{\mathcal{P}}^{m}, \widetilde{\mathcal{P}}^{m}+i \mathcal{P}^{m}, \widetilde{\mathcal{P}}^{m}-i \mathcal{P}^{m}\right\}$, where the linear combination of the tetrad vectors can be grouped by a single element as: $X^{\alpha}=a\left(\mathcal{P}^{m}\right)+b\left(\tilde{\mathcal{P}}^{m}\right)+c\left(\tilde{\mathcal{P}}^{m}+i \mathcal{P}^{m}\right)+$ $d\left(\tilde{\mathcal{P}}^{m}-i \mathcal{P}^{m}\right)$, the covariant derivatives in the $X^{\alpha}$ direction can be given by,

$X^{\alpha} \nabla_{a}=a\left(\mathcal{P}^{m} \nabla_{\mathrm{m}}\right)+b\left(\tilde{\mathcal{P}}^{m} \nabla_{m}\right)+c\left(\tilde{\mathcal{P}}^{m}+i \mathcal{P}^{m}\left(\nabla\left(\tilde{\mathcal{P}}_{m}+i \mathcal{P}_{m}\right)\right)\right)+c\left(\tilde{\mathcal{P}}^{m}-i \tilde{\mathcal{P}}^{m}\left(\nabla\left(\mathcal{P}_{m}-i \tilde{\mathcal{P}}_{m}\right)\right)\right)$

Denoting the tetrad indices as,

$$
\begin{array}{ccc}
\mathcal{P}^{m} & \rightarrow & l^{a} \\
\tilde{\mathcal{P}}^{m} & \rightarrow & n^{a} \\
\tilde{\mathcal{P}}^{m}+i \mathcal{P}^{m} & \rightarrow & m^{a} \\
\tilde{\mathcal{P}}^{m}-i \mathcal{P}^{m} & \rightarrow & \widetilde{m}^{a}
\end{array}
$$

Denoting the operators as, 


$$
\begin{array}{rccccc}
D & \rightarrow & \mathcal{P}^{m} \nabla_{\mathrm{m}} & \rightarrow & l^{a} \partial_{a} \\
\Delta & \rightarrow & \tilde{\mathcal{P}}^{m} \nabla_{m} & \rightarrow & n^{a} \partial_{a} \\
\delta^{\prime} & \rightarrow & \tilde{\mathcal{P}}^{m}+i \mathcal{P}^{m}\left(\nabla\left(\tilde{\mathcal{P}}_{m}+i \mathcal{P}_{m}\right)\right) & \rightarrow & m^{a} \partial_{a} \\
\bar{\delta} & \rightarrow & \tilde{\mathcal{P}}^{m}-i \tilde{\mathcal{P}}^{m}\left(\nabla\left(\mathcal{P}_{m}-i \tilde{\mathcal{P}}_{m}\right)\right) & \rightarrow & \tilde{m}^{a} \partial_{a}
\end{array}
$$

Here, we will notice one thing that, the time or present is actually a 2D rather than a stubborn 1D trajectory as because the rotation of time takes place by the generated time lines/CTC's of the time grids as seen in section 5: thus telling us that as in case of locally generated CTC's, the end point of past can meet the starting point of future which again meets the end points of the past and repeated continuation takes place through those grids) [48]. Therefore, due to rotation, its necessary to denote the spinorial indices of the tetrad as,

$$
\begin{aligned}
& \left.\begin{array}{rlll}
\kappa & := & & -m^{a} D l_{a} \\
\sigma & := & & -m^{a} \delta^{\prime} l_{a} \\
\tau & := & -m^{a} \Delta l_{a} \\
\rho & := & -m^{a} \bar{\delta} l_{a} \\
\pi & := & \widetilde{m}^{a} D n_{a} \\
\mu & := & \widetilde{m}^{a} \delta^{\prime} n_{a} \\
v & := & \widetilde{m}^{a} \Delta n_{a} \\
\varpi & := & \widetilde{m}^{a} \bar{\delta} n_{a} \\
\epsilon & := & -\frac{1}{2}\left(n^{a} D l_{a}-\widetilde{m}^{a} D m_{a}\right)
\end{array}\right\}\left|\begin{array}{cccc}
\kappa & \sigma & \tau & \rho \\
\pi & \mu & v & \varpi \\
\epsilon & \gamma & \beta & \alpha
\end{array}\right|_{4 \times 3} \\
& \epsilon:=-\frac{1}{2}\left(n^{a} D l_{a}-\widetilde{m}^{a} D m_{a}\right) \\
& \gamma:=-\frac{1}{2}\left(n^{a} \Delta l_{a}-\widetilde{m}^{a} \Delta m_{a}\right) \\
& \beta:=-\frac{1}{2}\left(n^{a} \delta^{\prime} l_{a}-\widetilde{m}^{a} \delta^{\prime} m_{a}\right) \\
& \left.\alpha:=\quad-\frac{1}{2}\left(n^{a} \bar{\delta} l_{a}-\tilde{m}^{a} \bar{\delta} m_{a}\right)\right)
\end{aligned}
$$

Thereby deriving the transportation/propagation vectors as in [46],

$$
\begin{aligned}
& \left.D l^{a}=(\epsilon+\tilde{\epsilon}) l^{a}-\tilde{\kappa} m^{a}-\kappa \tilde{m}^{a}\right) \\
& \Delta l^{a}=(\gamma+\tilde{\gamma}) l^{a}-\tilde{\gamma} m^{a}-\gamma \widetilde{m}^{a} \\
& \left.\delta^{\prime} l^{a}=(\tilde{\alpha}+\beta) l^{a}-\tilde{\rho} m^{a}-\sigma \tilde{m}^{a}\right\} l^{a} \\
& \left.\bar{\delta} l^{a}=(\alpha+\tilde{\beta}) l^{a}-\tilde{\sigma} m^{a}-\rho \tilde{m}^{\alpha}\right) \\
& \left.D n^{a}=\pi m^{a}+\tilde{\pi} \tilde{m}^{a}-(\epsilon+\epsilon) n^{a}\right) \\
& \Delta n^{a}=v m^{a}+\tilde{v} \tilde{m}^{a}-(\gamma+\tilde{\gamma}) n^{a} \\
& \left.\delta^{\prime} n^{a}=\mu m^{a}+\widetilde{\varpi} \widetilde{m}^{a}-(\tilde{\alpha}+\beta) n^{a}\right\} n^{a} \\
& \left.\bar{\delta} n^{a}=\varpi m^{a}+\tilde{\mu} \tilde{m}^{a}-(\alpha+\tilde{\beta}) n^{a}\right) \\
& \left.\begin{array}{rl}
D m^{a} & =(\epsilon-\tilde{\epsilon}) m^{a}+\tilde{\pi} l^{a}-\kappa n^{a} \\
\Delta m^{a} & =(\gamma-\tilde{\gamma}) m^{a}+\tilde{v} l^{a}-\tau n^{a} \\
\delta^{\prime} m^{a} & =(\beta-\tilde{\alpha}) m^{a}+\widetilde{\varpi} l^{a}-\sigma n^{a} \\
\bar{\delta} m^{a} & =(\alpha-\tilde{\beta}) l^{a}+\tilde{\mu} l^{a}-\rho n^{a}
\end{array}\right\} m^{a} \\
& D \widetilde{m}^{a}=(\tilde{\epsilon}-\epsilon) \tilde{m}^{a}+\pi l^{a}-\tilde{\kappa} n^{a} \\
& \Delta \widetilde{m}^{a}=(\tilde{\gamma}-\gamma) \tilde{m}^{a}+v l^{a}-\tilde{\tau} n^{a} \\
& \left.\delta^{\prime} \tilde{m}^{a}=(\beta-\tilde{\alpha}) \tilde{m}^{a}+\mu l^{a}-\tilde{\rho} n^{a}\right\} \tilde{m}^{a} \\
& \left.\bar{\delta} \widetilde{m}^{a}=(\alpha-\tilde{\beta}) \tilde{m}^{a}+\varpi l^{a}-\tilde{\sigma} n^{a}\right)
\end{aligned}
$$


The $\widetilde{m}^{a} \approx \tilde{\mathcal{P}}^{m}-i \mathcal{P}^{m}$ denotes the complex time in the tunneling phase of the singularity where the singularity being a point in time is smoothen away by the imaginary values as mentioned by Hawking [47] and in [48]. We have showed this tunneling with the help of a spherinder solutions. If in the equation $(\epsilon+\tilde{\epsilon}) l^{a}-$ $\tilde{\kappa} m^{a}-\kappa \widetilde{m}^{a}$, the $(\epsilon+\tilde{\epsilon})=0$ and $\kappa=0$ then $D l^{a}$ denotes the $l^{a}$ to be tangent to an affine parameterized geodesic where as mentioned before, $l^{a}=\mathcal{P}^{m}$ showing the arrow of the local/true time from past to future via present over an embedded 2D nonlocal timeline. The same is applicable for the other equations.

\section{Discussions}

We have shown the formation of the WH without any exotic matter by modifying the Throne-Morris $\mathrm{WH}$ metric by $f(R, T)$ theories of gravity where it has been explicitly mentioned and shown the numerous temporal intervals that a traveler would have come through if he/she tries to cross the WH from one station to another. This accompanied by 9 poles with the validity of 2 poles shows the fact that the WH can not only be traversed but also its possible to tunnel out the ring singularities by a complex null NP-tetrad thereby providing a viable source for the temporal arrow to be of 2D that accompanies with $3265824 d-4$ spatial sheath that makes the time traveler inside the WH look after 96 unique solutions of spacelike trajectories of his timelines and 3265824 scattered trajectories of his timeline through a one way superficially visible hypermembrane (or sheath) that will rule out all sorts of paradoxes associated with the time travel. Three key points needed to be mentioned as a viability of this paper - (i). The formation of a naked singularity, (ii). The inclusion of $\mathrm{f}(\mathrm{R}, \mathrm{T})$ modified gravity approach to rule out the presence of exotic matters where all forms of energy conditions, SEC, WEC, NEC, DEC, ANEC are greater than or equal to 1, (iii). The smoothen out of the singularity through imaginary time and tunneling the WH throat interval $\left[\left.\frac{+\ell-\ell}{2+\delta}\right|_{(\mathrm{Up})},\left.\frac{+\ell-\ell}{2+\delta}\right|_{(\mathrm{down})}\right]$ with the help of a spherinder that in practical can form the complex notion of the timelike trajectories as represented through the Neumann-Penrose tetrad formalisms.

\section{References}

1. Rindler, W. (2001). Time from Newton to Einstein to Friedman. KronoScope, 1(1), 63-73. https://doi.org/10.1163/156852401760060928

2. Trautman, A. (2002). Lectures on General Relativity. General Relativity and Gravitation, 34(5), 721-762. https://doi.org/10.1023/a:1015939926662

3. Bhattacharjee, D. Path Tracing Photons Oscillating Through Alternate Universes Inside a Black Hole. Preprints 2021, 2021040293, 1-3. https://dx.doi.org/10.20944/preprints202104.0293.v1

4. Bhattacharjee, D. Deciphering Black Hole Spin, Inclination angle \& Charge From Kerr Shadow. Preprints 2021, 2021040315, 1-3. https://dx.doi.org/10.20944/preprints202104.0315.v1

5. Bhattacharjee, D. (2020d). Solutions of Kerr Black Holes subject to Naked Singularity and Wormholes. Authorea, 1-4. https://doi.org/10.22541/au.160693414.46356832/v1

6. Wells, H. G. (2021). The Time Machine (Royal Collector's Edition) (Case Laminate Hardcover with Jacket). Royal Classics.

7. Sagan, C. (2019). Contact: A Novel (Reprint ed.). Gallery Books.

8. Nolan, C. (Director). Interstellar [Flim], Paramount Pictures, Warner Bros. Pictures, Legendary Pictures, Syncopy, Lynda Obst Productions.

9. Vasiliev, V. V., \& Fedorov, L. V. (2018). To the Schwarzschild Solution in General Relativity. Journal of Modern Physics, 09(14), 2482-2494. https://doi.org/10.4236/jmp.2018.914160

10. Katanaev, M. O. (2014). Passing the Einstein-Rosen bridge. Modern Physics Letters A, 29(17), 1450090. https://doi.org/10.1142/s0217732314500904

11. Torii, T., \& Shinkai, H. A. (2013). Wormholes in higher dimensional space-time: Exact solutions and their linear stability analysis. Physical Review D, 88(6). https://doi.org/10.1103/physrevd.88.064027 
12. Hawking, S. W., Ellis, G. F. R., Landshoff, P. V., Nelson, D. R., Sciama, D. W., \& Weinberg, S. (1975). The Large Scale Structure of Space-Time (Cambridge Monographs on Mathematical Physics) (New Ed). Cambridge University Press.

13. Morris, M. S., \& Thorne, K. S. (1988). Wormholes in spacetime and their use for interstellar travel: A tool for teaching general relativity. American Journal of Physics, 56(5), 395-412. https://doi.org/10.1119/1.15620

14. Mars, M. (1999). A spacetime characterization of the Kerr metric. Classical and Quantum Gravity, 16(7), 2507-2523. https://doi.org/10.1088/0264-9381/16/7/323

15. Bhattacharjee, D. Positive Energy Driven CTCs In ADM 3+1 Space - Time of Unprotected Chronology. Preprints 2021, 2021040277, 1-7. https://dx.doi.org/10.20944/preprints202104.0277.v1

16. James, O., von Tunzelmann, E., Franklin, P., \& Thorne, K. S. (2015). Visualizing Interstellar's Wormhole. American Journal of Physics, 83(6), 486-499. https://doi.org/10.1119/1.4916949

17. Hawking, S. W. (1992). Chronology protection conjecture. Physical Review D, 46(2), 603-611. https://doi.org/10.1103/physrevd.46.603

18. Luminet, J. P. (2018). Closed Timelike Curves and Singularities. Inference: International Review of Science, 4(1). https://doi.org/10.37282/991819.18.32

19. Throne, K. S. (1993). Closed Timelike Curves. A Caltech Goldenrod Preprint in Theoretical Physics or Gravitational Physics.

20. Chanda, A. Dey, S. Paul, B. C. (2021). Morris-Thorne Wormholes in $f(R, T)$ modified theory of gravity arXiv:2102.01556v1 [gr-qc]

21. Stewart, I. (2010). Grandfather paradox. Nature, 464(7293), 1398. https://doi.org/10.1038/4641398a

22. Davis, M. (2020, December 23). 7. Polchinski's paradox. Big Think. https://bigthink.com/surprisingscience/10-bizarre-paradoxes?rebelltitem $=8$

23. Christoforou, P. (2019, December 1). Time Travel \& the Bootstrap Paradox Explained. https://www.astronomytrek.com/the-bootstrap-paradox-explained/

24. Perry, P. (2018, October 5). There are 2 dimensions of time, theoretical physicist states. Big Think. https://bigthink.com/philip-perry/there-are-in-fact-2-dimensions-of-time-one-theoretical-physiciststates

25. Ewbank, A. (2018, December 24). When Stephen Hawking Threw a Cocktail Party for Time Travelers. Atlas Obscura. https://www.atlasobscura.com/articles/stephen-hawking-time-travelers-party

26. Spherinder. (2021, May 30). In Wikipedia. https://en.wikipedia.org/wiki/Spherinder

27. Landau, L. D. (2021). The Classical Theory of Fields (Course of Theoretical Physics) by L. D. Landau (1-Jan-1980) Paperback. Butterworth-Heinemann; Revised edition (1 Jan. 1980).

28. Rezzolla, L., \& Zanotti, O. (2018). Relativistic Hydrodynamics (Reprint ed.). Oxford University Press.

29. Visser, M (2007). "The Kerr spacetime: A brief introduction". p. 15, Eq. 60-61, p. 24, p. 35. arXiv:0706.0622v3[gr-qc].

30. Boyer, R. H., \& Lindquist, R. W. (1967). Maximal Analytic Extension of the Kerr Metric. Journal of Mathematical Physics, 8(2), 265-281. https://doi.org/10.1063/1.1705193

31. Carter, B. (1968). Global Structure of the Kerr Family of Gravitational Fields. Physical Review, 174(5), 1559-1571. https://doi.org/10.1103/physrev.174.1559

32. Bardeen, J. M., Press, W. H., \& Teukolsky, S. A. (1972). Rotating Black Holes: Locally Nonrotating Frames, Energy Extraction, and Scalar Synchrotron Radiation. The Astrophysical Journal, 178, 347. https://doi.org/10.1086/151796

33. Godani, N., \& Samanta, G. C. (2019). Static traversable wormholes in $f(R, T)=R+2 \alpha \ln T$ gravity. Chinese Journal of Physics, 62, 161-171. https://doi.org/10.1016/j.cjph.2019.09.009

34. Kerr metric. (2021, April 30). In Wikipedia. https://en.wikipedia.org/wiki/Kerr_metric 
35. Sahoo, P. K., Moraes, P. H. R. S., \& Sahoo, P. (2018). Wormholes in $\$ \$ R^{\wedge} 2 \$ \$$ R 2 -gravity within the $f(R, T)$ formalism. The European Physical Journal C, 78(1). https://doi.org/10.1140/epjc/s10052018-5538-1

36. McMahon, D. (2008). String Theory Demystified (1st ed.). McGraw-Hill Education.

37. Bhattacharjee, D., Harikant, A., \& Singha Roy, S. (2019b). Improvising the Hierarchy Problem with respect to F-Theory. ResearchGate, 1-3. https://doi.org/10.13140/RG.2.2.35954.53444

38. NineDimensionalBeing. (2015, March 3). Exploring the 4D Spherinder. Imgur. https://imgur.com/gallery/ORY5G/comment/374041029/1

39. Newman-Penrose formalism. (2021, January 4$). \quad$ In $\quad$ Wikipedia. https://en.wikipedia.org/wiki/Newman\%E2\%80\%93Penrose_formalism

40. Newman, E., \& Penrose, R. (1962). An Approach to Gravitational Radiation by a Method of Spin Coefficients. Journal of Mathematical Physics, 3(3), 566-578. https://doi.org/10.1063/1.1724257

41. Newman, E., \& Penrose, R. (1962b). An Approach to Gravitational Radiation by a Method of Spin Coefficients. Journal of Mathematical Physics, 3(3), 566-578. https://doi.org/10.1063/1.1724257

42. Chandrasekhar, L. T. S. (1998). The Mathematical Theory of Black Holes (Oxford Classic Texts in the Physical Sciences). Clarendon Press.

43. Griffiths, J. B. (2012). Exact Space-Times in Einstein's General Relativity (Cambridge Monographs on Mathematical Physics) (1st ed.). Cambridge University Press.

44. Frolov, \& Novikov. (1998). Black Hole Physics: Basic Concepts and New Developments (Fundamental Theories of Physics, 96) (1998th ed.). Springer.

45. Ashtekar, A., Fairhurst, S., \& Krishnan, B. (2000). Isolated horizons: Hamiltonian evolution and the first law. Physical Review D, 62(10). https://doi.org/10.1103/physrevd.62.104025

46. O'Donnell, P. (2003) Introduction to 2-Spinors in General Relativity. Singapore: World Scientific.

47. Hawking, S. W. (2001). The Universe in a Nutshell (1st ed.). Bantam.

48. Bhattacharjee, D. The Gateway to Parallel Universe \& Connected Physics. Preprints 2021, 2021040350, 1-20, https://dx.doi.org/10.20944/preprints202104.0350.v1 